\title{
An Active Contour-Based Atlas Registration Model Applied to Automatic Subthalamic Nucleus Targeting on MRI: Method and Validation
}

\author{
Valérie Duay $^{1}$, Xavier Bresson ${ }^{2}$, Javier Sanchez Castro ${ }^{1}$, Claudio Pollo ${ }^{3}$, \\ Meritxell Bach Cuadra ${ }^{1}$, and Jean-Philippe Thiran ${ }^{1}$ \\ ${ }^{1}$ Signal Processing Laboratory (LTS5), EPFL, CH-1015 Lausanne \\ ${ }^{2}$ UCLA Departement of Mathematics, Los Angeles, CA 90095 \\ ${ }^{3}$ Department of Neurosurgery, University Hospital (CHUV), CH-1011 Lausanne
}

\begin{abstract}
This paper presents a new non parametric atlas registration framework, derived from the optical flow model and the active contour theory, applied to automatic subthalamic nucleus (STN) targeting in deep brain stimulation (DBS) surgery. In a previous work, we demonstrated that the STN position can be predicted based on the position of surrounding visible structures, namely the lateral and third ventricles. A STN targeting process can thus be obtained by registering these structures of interest between a brain atlas and the patient image. Here we aim to improve the results of the state of the art targeting methods and at the same time to reduce the computational time. Our simultaneous segmentation and registration model shows mean STN localization errors statistically similar to the most performing registration algorithms tested so far and to the targeting expert's variability. Moreover, the computational time of our registration method is much lower, which is a worthwhile improvement from a clinical point of view.
\end{abstract}

\section{Introduction}

Deep brain stimulation of the subthalamic nucleus (STN) has revealed to be the most effective surgical technique for the treatment of Parkinson's disease or other movement disorders [1. The STN is a very small brain structure not easy to locate due to its bad visibility in common medical imaging modalities such as MR images. Therefore, atlas-based segmentation methods are often considered to estimate its position in the patient's image. In our previous work 234, we first evaluated the usability of different atlas-based and nonrigid registration algorithms to locate the STN automatically. Then, we showed that the position of the STN is spatially influenced by the position of the lateral and third ventricles (Fig. 1(a)]. To demonstrate that, we registered the binary masks of these structures of interest after having previously delineated them in the atlas and in the patient image. Some efforts for automatically estimating the STN target have been reported recently (see [5] for example). They construct the

D. Metaxas et al. (Eds.): MICCAI 2008, Part II, LNCS 5242, pp. 980-988, 2008.

(C) Springer-Verlag Berlin Heidelberg 2008 
STN ground truth through indirect methods by registering anatomical, histological, and/or electrophysiological atlases with the reference patient, model or template but, as far as we know, our study is the first one that constructs the ground truth in a direct way from visible STNs and that directly studies the influence of neighboring structures in the estimation of the STNs location. In this paper, we present and evaluate the performance of a new atlas registration method that permits to register selected structures (here the lateral and third ventricles) without needing a previous segmentation of the patient image. Our model was specially designed to be very flexible to incorporate a large variety of constraints in the registration process and thus to be easily adaptable to most of the atlas-based segmentation problems (not only to the STN targeting problem addressed here). The final goal of this work is to provide a registration model that is not only more accurate than the state of the art methods but also that has a lower computational time.

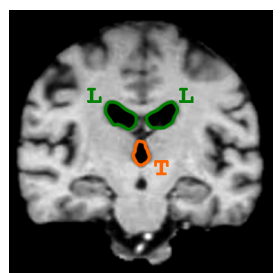

(a)

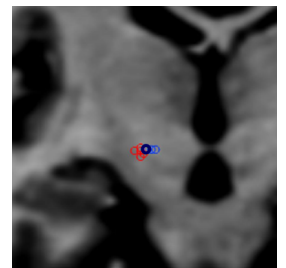

(b)

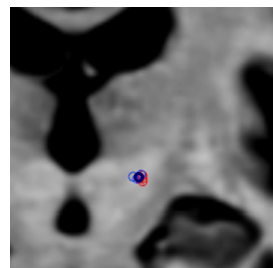

(c)

Fig. 1. Left and right STN atlases (coronal views). a) Structures selected in the atlas to estimate the position of the STN: lateral ventricles (L) and third ventricle $(\mathrm{T}) . \mathrm{b}$ ), c) Left and right STN ground truth (dark blue) computed from the estimations of two medical experts (Expert 1 in red and Expert 2 in light blue). Each STN position is represented by a circle of radius $1 \mathrm{~mm}$ (this model seems reasonable considering the very small size of the STN).

Our novel registration model aims to combine the advantages of the dense deformation field computed by non rigid registration algorithms with local segmentation constraints defined in the Active Contour (AC) framework. The dense deformation field computed from the registration of visible image contours allows to easily estimate the position of structures with not well or without visible contours in the image such as the STN. The AC segmentation model is often able to delineate visible boundaries in a more accurate way than the segmentation method based on atlas registration. This is due to their global and local matching criteria that can exploit the image information directly linked to the object to be extracted.

The main source of inspiration of our joint registration and segmentation algorithm is the partial derivative equation (PDE)-based method proposed by 
Vemuri et al. in [6] 1 . The formulation of their model is intuitively deduced from the general level set evolution equation (10) introduced by Osher and Sethian in 8.

$$
\frac{\partial \phi(x, t)}{\partial t}=\phi_{t}(x, t)=\nu(\phi(x, t))|\nabla \phi(x, t)|,
$$

where $\nu$ is the velocity of the flow or speed function that contains the local segmentation and contour regularization constraints and $\phi$ is the level set function used to represent implicitly the active contour. In [8], the active contour is modeled by the zero level of the well known signed distance function. The original idea brought by Vemuri's model is to use as $\phi$, the intensity function of the image to be registered (the moving image). Thus, the level sets considered in the segmentation process correspond to the contours naturally present in the moving image. A dense deformation field is then generated by tracking the deformation of these level sets during the segmentation process. The main advantage of this model is the ability of registering any type of contours (closed, open, connected or disconnected) unlike the signed distance function that can only model closed and disconnected contours. However, this advantage can also be a drawback. Since all the level sets of the reference image are considered, content-based inconsistencies between both images, e.g. a lesion in the patient image, can lead to misregistration. Moreover, since this contour representation does not permit to select consistent contours or closed regions in the atlas, the Vemuri's model is limited to pixel-based segmentation forces only. That means that this model cannot use in the registration process typical segmentation forces of the AC framework such as boundary-based and region-based forces (see Section 2.2). Unlike [6], our registration model is able to use forces developed in the $\mathrm{AC}$ framework since it is based on the general level set approach 8. Moreover, in [9] we propose to handle the registration of multiple regions by modeling the active contours with a label function.

This paper is organized as follows. In Section 2, we present an overview of our non parametric atlas registration framework. Then, we evaluate in Section 3 our registration method to the challenging STN targeting problem. We use a statistical test to compare our results with state of the art registration algorithms. Finally, results are discussed and conclusions are drawn in Section 4

\section{Active Contour-Based Registration Model}

\subsection{Deformation Field Extraction}

The general formulation of our model is derived from the tracking of the signed distance function motion with the optical flow (OF) approach [10. The OF technique assumes that the brightness of the moving image, here the level set

\footnotetext{
${ }^{1}$ There exists also a variational energy-based approach initiated by Yezzi et al. in 7. We chose the PDE-based approach because it seems more flexible to solve joint registration and segmentation problems notably in the choice of the attractive and regularization terms composing the speed function.
} 
function $\phi$, stays constant for small displacements and a short period of time: $\phi(x, t)=\phi(x+d u, t+d t) \Rightarrow d \phi(x, t)=0$, where $d u$ is the instantaneous deformation vector field and $d \phi$ is the total derivative of $\phi$. By using the chain rule, this OF constraint can be rewritten as the evolution equation of a vector flow:

$$
\frac{\partial u(x, t)}{\partial t}=-\frac{\phi_{t}(x, t)}{|\nabla \phi(x, t)|} \frac{\nabla \phi(x, t)}{|\nabla \phi(x, t)|},
$$

where $\phi_{t}$, given by (1), represents the variation of the level set function according to the desired forces such as supervised segmentation, shape prior knowledge or contour regularization. Thus, by introducing the evolution equation of the level set segmentation model (1) in (2), we obtain the following formula merging the active contour segmentation framework with the image registration task:

$$
\frac{\partial u(x, t)}{\partial t}=-\nu(\phi(x, t)) \frac{\nabla \phi(x, t)}{|\nabla \phi(x, t)|} .
$$

The level set function $\phi$ does not evolve with the usual finite difference scheme. Its position at time $t$ is given by the deformation field $u(x, t)$ and the initial level set function $\phi(x, 0)$ such that: $\phi(x, t):=\phi(x+u(x, t), 0)$, with $\phi(x, 0)$ is the initial active contour position. This ensures that the evolution of the level set function exactly corresponds to the current deformation. Thus, (3) becomes:

$$
\frac{\partial u(x, t)}{\partial t}=-\nu(\phi(x+u(x, t), 0)) \frac{\nabla \phi(x+u(x, t), 0))}{\mid \nabla \phi(x+u(x, t), 0)) \mid} .
$$

This equation is the general formulation of our active-contour atlas registration model. It defines a displacement vector (or registration force) at each point of the level set function. The level set function $\phi$ models the contours of the objects selected in the atlas to drive its registration. For the STN targeting application, we use a label function as $\phi$. See [9] for more details on the adaptation of our model to this active contour representation.

\subsection{Segmentation / Registration Forces}

A large variety of forces coming from the $\mathrm{AC}$ segmentation framework can be used in our simultaneous segmentation and registration model (see Figure 2). For the STN surgical targeting application we address in this paper, we propose to use a registration force based on mean priors inspired by the region-based segmentation model proposed by Chan and Vese [11. This force is derived from an energy designed to be minimal when the mean of a region $\Omega$ defined in the target image by the evolving level set function is close to the mean of the corresponding region in the reference image: $E=\int_{\Omega_{\text {in }}}\left|I(x)-\mu_{\text {in }}^{\text {prior }}\right|^{2} d x+\int_{\Omega_{\text {out }}} \mid I(x)-$ $\left.\mu_{\text {out }}^{\text {prior }}\right|^{2} d x$, where $\Omega_{\text {in }}$ and $\Omega_{\text {out }}$ are respectively the image area inside and outside the active contour, $\mu_{\text {prior }}$ is the prior mean of a given region extracted from a reference image (the atlas) and $I$ is the intensity function of the image to be segmented. The derived speed function is: $\nu=\left(I(x)-\mu_{\text {in }}^{\text {prior }}\right)^{2}+\left(I(x)-\mu_{\text {out }}^{\text {prior }}\right)^{2}$. 


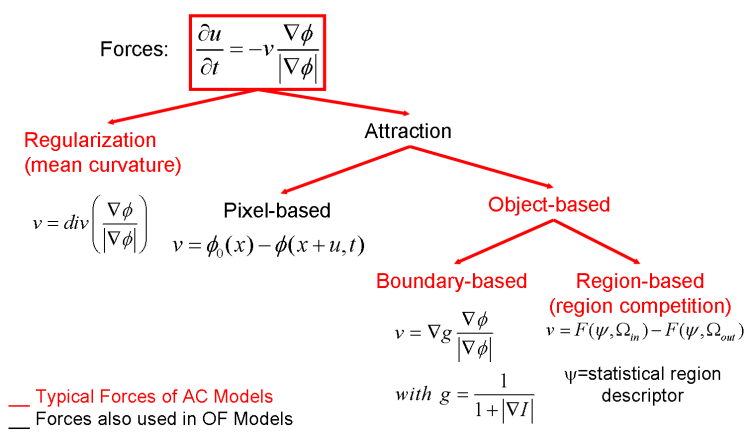

Fig. 2. Classification of the AC forces according to their effect in a contour matching process. 1) The regularization mean curvature forces smooth the level sets by minimizing their length. 2) The pixel-based forces (typical forces of the OF model) are based on the smallest image feature, the pixel value. They can match any type of contours (open or closed) but are very sensitive to image noise and limited to recover small deformations. 3) The object-based forces can register image regions (closed contours). If we apply an object-based force on each point of a signed distance function, every level set will collapse to the closest target contour in the target image. Therefore, they need to be computed only on the contours modeled by the level set function $\phi$. The region-based forces are much less sensitive to noise than the boundary-based forces.

This mean-based force assumes that corresponding regions between the reference and the target images have similar means. Note that $\mu^{\text {prior }}$ does not evolve during the registration process. Hence it is only computed once on the reference image in a pre-process step. At each iteration, the displacement computed on the active contour is propagated to the whole image by linear diffusion. Then, the transformation is constrained to be bijective following a technique inspired by Thirion et al. [12] and described in [9]. Finally, the registration process is speeded up with a multi-resolution approach.

\section{Automatic Subthalamic Nucleus Targeting for Deep Brain Stimulation Surgery}

\subsection{Data Set}

In this study, a set of 39 bilaterally implanted parkinsonian patients were considered (78 STNs). Two kinds of images were acquired pre-operatively for each patient: 3D T1- weighted MPRAGE MRI sequence (Siemens Vision, 1.5T, Erlangen, Germany) TR $9.7 \mathrm{~ms}$, TE $4 \mathrm{~ms}$, number of slices/slice thickness: 164/1.40 mm, FOV 280x280, matrix 256x256, pixel size 1.09x1.09 mm and few coronal slices of an IR T2-weighted, TR 2,560 ms, TE $4 \mathrm{~ms}$, number of slices/slice thickness: $7 / 3 \mathrm{~mm}$, FOV 300x300, matrix 512x512, pixel size 0.59x0.59 mm. Taking profit from the fact that in some specific patients the STN is visible in MR T2weighted images a ground truth is constructed from expert's knowledge (Fig. 
1(b) 1(c) following the protocol described in [2344. Finally, 8 patients with clearly visible STNs were selected to take part in this validation process. Among the 8 selected patients (16 STNs), the experts have selected the one with the most clearly visible STN as a reference subject (atlas), both for the right and left sides.

\subsection{Registration Methods}

The following registration methods have been compared through the validation scheme proposed in 234. The three following ones perform the registration of the whole left and right STN atlases: Affine: A 12 degrees of freedom (translation, rotation, scaling and shearing) mutual-information-based algorithm based on the work of Maes et al. 13] which has also been applied as a pre-alignment step for the non-rigid registration algorithms described below. All these algorithms have been used with a bijectivity scheme. Demons: It is an independent implementation of the intensity-based algorithm developed by Thirion [12]. BSplines: It is a mutual-information-based free-form deformation algorithm similar to the method proposed by Rueckert et al. [14]. The three following local non rigid registration methods have been used to register the structures of interest (the lateral and third ventricles) only: Segm D.: The demons registration is applied between the binary masks of the structures considered both for the atlas and the patient under study. This is the algorithm we used in [3] to determine which structures influence the STN position. RBF: In this mutual information-based technique, the deformation that registers the intensity atlas onto the patient image is modeled with a linear combination of radial basis functions with finite support that are placed on relevant image point features [15]. $A C$ : Our active contour-based atlas registration model we described in Section 2.

\subsection{Results}

The estimation errors have been computed as the Euclidean distance from the estimated target given by each method to the ground truth. The inter-expert variability has been computed as the Euclidean distance from the expert targeting to the ground truth. The means and std of the errors committed when applying each of the methods and the expert variability are shown in Table 1 They are ordered by decreasing mean error. To evaluate significant differences, we have performed as in [16] a one-way analysis of variance (ANOVA) statistical test. This test allows to evaluate the hypothesis that the registration errors and the expert variability come from distributions with equal means at a $5 \%$ significance level. In Fig. 3. a statistical box plot generated by this test is shown as well as the result of a multi-comparison test of the means. Fig. 3(b) well shows that the Demons algorithm applied on segmented structures, the BSplines algorithm and our model have a mean error that is statistically different from the affine registration method and similar to the targeting expert's variability. Concerning the computation time, the average time of the algorithms registering the whole 
Table 1. Statistical measures: means and std of the estimation errors

\begin{tabular}{|c||c|}
\hline Methods and Experts & mean \pm std $[\mathrm{mm}]$ \\
\hline \hline Affine & $2.42 \pm 0.84$ \\
RBF & $1.83 \pm 0.53$ \\
Demons & $1.77 \pm 0.65$ \\
AC & $\mathbf{1 . 7 4} \pm \mathbf{0 . 5 5}$ \\
BSplines & $1.72 \pm 0.48$ \\
Expert 1 & $1.61 \pm 0.29$ \\
Segm Demons & $1.58 \pm 0.71$ \\
Expert 2 & $1.40 \pm 0.38$ \\
\hline
\end{tabular}

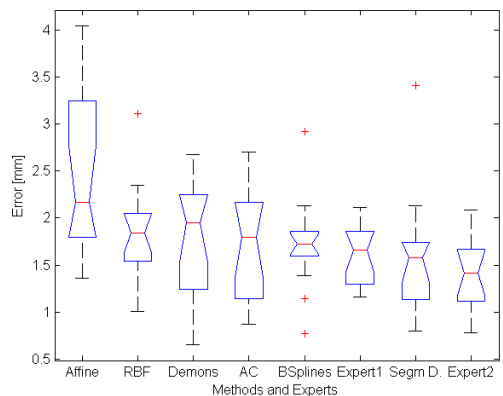

(a)

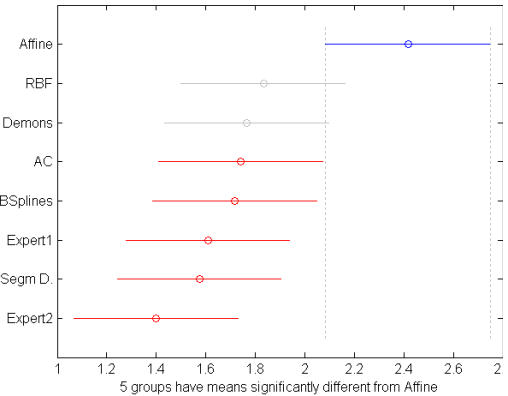

(b)

Fig. 3. Statistical tests of the errors committed by the different registration methods and medical experts

STN atlas (BSplines, Demons) is about 90 minutes and the one of the local non rigid registration methods ( $\mathrm{RBF}$, Segm Demons, $\mathrm{AC}$ ) is about 25 minutes 2 .

\section{Discussion}

In this paper, we show that the targeting variability of our novel active contourbased atlas is not larger than the one of the medical experts and that its mean registration error is statistically similar to the Demons algorithm applied on segmented structures and the BSplines algorithm. The two main advantages of our algorithm is that it is 4 to 5 times faster than the BSplines algorithm because it computes its registration forces only on structures of interest and it does not require a previous segmentation of the patient image as the Demons algorithm applied on segmented structures. As the estimation of the STNs location is mainly influenced by neighboring structures, the accuracy of the results is highly depending on the interpolation method used. The interpolation methods of image-based registration algorithms as our model or the BSplines and Demons algorithms present thus a drawback because of their arbitrary nature. The biomechanical algorithms are especially known for their interpolation model

${ }^{2}$ These times are related to a computer with the following characteristics: Intel(R) Pentium(R), 4 CPU, 2.8 GHz, 1.00 GB of Ram. 
based on physical laws of the tissues deformability. However these algorithms can not take into account all the forces that can interact with the moving image and it often requires the arbitrary setting of a rather large number of parameters. Future work involves to include in our active contour-based registration model a new type of segmentation / registration force derived from a prior deformation field model as the one proposed in [17. We would like to test if such prior knowledge will make our model more accurate than the most performing state of the art methods to estimate the position of the STN. This interpolation problem of the ventricles deformation has a large impact on the DBS success. Indeed, we estimate to $2 \mathrm{~mm}$ the distance we can have between benefic or undesirable DBS effects. Besides, an automatic pre-operative targeting with a STN localization error statistically similar to the expert's variability would be very interesting because following our neurosurgical clinical experience (more than 150 DBS surgeries), the final electrodes were implanted in about $2 / 3$ of the cases in the target positions manually selected in radiology [18.

\section{References}

1. Perlmutter, J., et al.: Deep brain stimulation. Annual Review of Neuroscience 29, 229-257 (2006)

2. Sanchez Castro, F.J., et al.: A cross validation study of deep brain stimulation targeting: From experts to atlas-based, segmentation-based and automatic registration algorithms. TMI 25(11) (2006)

3. Sanchez Castro, F.J., et al.: Feature-segmentation-based registration for fast and accurate deep brain stimulation targeting. In: CARS (2006)

4. Sanchez Castro, F.J., et al.: Automatic subthalamic nucleus targeting for deep brain stimulation. a validation study. In: CARS (2006)

5. D'Haese, P.F., et al.: Automatic selection of DBS target points using multiple electrophysiological atlases. In: Duncan, J.S., Gerig, G. (eds.) MICCAI 2005. LNCS, vol. 3750, pp. 427-434. Springer, Heidelberg (2005)

6. Vemuri, B.C., et al.: Image registration via level-set motion: Applications to atlasbased segmentation. MIA 7(1), 1-20 (2003)

7. Yezzi, A., et al.: A variational framework for joint segmentation and registration. In: MMBIA, pp. 44-51 (2001)

8. Osher, S., Sethian, J.A.: Fronts propagating with curvature-dependent speed algorithms based on hamilton-jacobi formulations. J. of Comp. Physics 79(1), 12 49 (1988)

9. Duay, V.: Deformation field estimation for atlas registration using the active contour framework. THÈSE NO 3979, EPFL (2007)

10. Barron, J.L., et al.: Performance of optical flow techniques. Intl. J. Comput. Vision 1, 43-77 (1994)

11. Chan, T.F., Vese, L.: Active contours without edges. IEEE Trans. Im. Proc. 10(2), 266-277 (2001)

12. Thirion, J.P., et al.: Image matching as a diffusion process: an analogy with maxwells demons. MIA 2(3), 243-260 (1998)

13. Maes, F., et al.: Multiphase multimodality image registration by maximization of mutual information. TMI 16(2), 187-198 (1997) 
14. Rueckert, D., et al.: Nonrigid registration using free-form deformations: Application to breast MR images. TMI 18(8), 712-721 (1999)

15. Sanchez Castro, F.J., et al.: Nonrigid medical image registration: Algorithms, validation and applications. THÈSE NO 3817, EPFL (2007)

16. Hellier, P., et al.: Retrospective evaluation of inter-subject brain registration. TMI 22(9), 1120-1130 (2003)

17. Charpiat, G., et al.: Shape statistics for image segmentation with prior. In: CVPR, pp. 1-6 (2007)

18. Pollo, C., et al.: Localization of electrodes in the subthalamic nucleus on magnetic resonance imaging. J. Neurosurg. 106(1), 36-44 (2007) 